\title{
Tratamiento estomatológico interdisciplinario del lupus eritematoso generalizado. Presentación de un caso
}

\section{RESUMEN}

El lupus eritematoso generalizado es una enfermedad crónica autoinmunitaria del tejido conectivo. Su compromiso multisistémico convierte a los pacientes que lo padecen en individuos de alto riesgo para el tratamiento estomatológico, por lo que se deben tener consideraciones individualizadas para su atención. Estomatológicamente en 2 a 80\% de los pacientes se observan úlceras orales, enfermedad periodontal, candidiasis oral, mucositis por inmunodepresores, síndrome de Sjögren y predisposición a infecciones. Reportamos el tratamiento estomatológico de una niña con lupus eritematoso generalizado y caries dental con el objetivo de dar a conocer las consideraciones interdisciplinarias, específicas e individualizadas antes, durante y después del tratamiento.

Palabras clave: lupus eritematoso generalizado, manifestaciones y consideraciones estomatológicas.

\section{Interdisciplinary dental treatment of lupus erythematosus. A case report}

\begin{abstract}
Lupus erythematosus is a chronic autoimmune disease, which involves connective tissue. The multisystem commitment transforms to lupus erythematosus patients in high-risk individuals for dental treatment, so that must be individualized considerations for your attention. In oral cavity is observed in $2 \%$ to $80 \%$ of the patients oral ulcers, periodontal disease, oral thrush, immunosuppressive mucositis, Sjögren's syndrome and susceptibility to infections.
\end{abstract}

We report dental treatment in a girl with lupus erythematosus and dental decay, aiming to report specific and individualized interdisciplinary considerations before, during and after dental treatment.

Key words: lupus erythematosus, oral and dental considerations.
Paola Gómez-Contreras ${ }^{1}$ Eduardo De la Teja-Ángeles ${ }^{2}$ Hilda Ceballos-Hernández ${ }^{3}$ Gerardo Elías-Madrigal ${ }^{3}$ Emely Estrada-Hernández ${ }^{3}$ Alonso Gutiérrez-Hernández ${ }^{4}$

${ }^{1}$ Residente de segundo año de la Especialidad de Estomatología Pediátrica.

2 Jefe del Servicio de Estomatología y profesor titular. ${ }^{3}$ Médico adscrito y profesor del Servicio de Estomatología Pediátrica.

${ }^{4}$ Médico Alergólogo e Inmunólogo. Pediatra adscrito al Servicio de Inmunología.

Instituto Nacional de Pediatría, Ciudad de México.

Recibido: 7 de marzo del 2013

Aceptado: 3 de febrero del 2015

Correspondencia: Dr. Eduardo De la Teja-Ángeles Instituto Nacional de Pediatría Insurgentes sur 3700-C

Insurgentes Cuicuilco, México, D.F.

Tel.: 10840900 ext. 1226

Este artículo debe citarse como Gómez-Contreras P, De la Teja-Ángeles E, CeballosHernández H, Elías-Madrigal G, Estrada-Hernández E, Gutiérrez-Hernández A. Tratamiento estomatológico interdisciplinario del lupus eritematoso generalizado. Presentación de un caso. Acta Pediatr Mex 2015;36:330-336. 


\section{INTRODUCCIÓN}

El lupus eritematoso generalizado es un padecimiento que causa lesiones multisistémicas por mecanismos autoinmunitarios desencadenados por agresiones ambientales en presencia de genes susceptibles. ${ }^{1-4}$ Es mediado por complejos inmunitarios que producen autoanticuerpos, mismos que reaccionan con constituyentes celulares, como el ADN, provocando una respuesta inflamatoria y alteraciones en el funcionamiento de ciertos órganos. ${ }^{2-4}$ Sus manifestaciones clínicas difieren en cada paciente y para confirmar su diagnóstico deben de existir al menos cuatro de los once criterios señalados por la Academia Americana de Reumatología ${ }^{1,4}$ (Cuadro 1).

La etiología exacta del lupus eritematoso generalizado es desconocida; sin embargo, existen factores que pueden estar involucrados como predisposición genéticas, radiaciones ultravioleta, infecciones por citomegalovirus o
Epstein-Barr, medicamentos antihipertensivos o anticonvulsivos y cambios hormonales..$^{2,3}$ Tiene una prevalencia de 1 en 2000 en caucásicos y de 1 en 250 afroamericanos, asiáticos e hispanos. ${ }^{1}$ La incidencia varía de 1.8 a 7.6 casos por cada 100000 personas por año. En los adultos predomina en el sexo femenino con $90 \%$ de los casos. ${ }^{1}$ Entre 20 y $30 \%$ de los casos se inicia en la edad pediátrica y algunos autores relacionan su exacerbación con factores climáticos. ${ }^{1}$ Su tratamiento consiste en antiinflamatorios esteroides (prednisona) y no esteroides (AINES); ${ }^{1}$ otros medicamentos empleados son antimaláricos (hidroxocloroquina) e inmunodepresores como azatioprina, metotrexato, ciclofosfamida, clorambucilo y micofenolato. ${ }^{1,4}$ Las manifestaciones estomatológicas se observan en 2 a $80 \%$ de los pacientes con una prevalencia de 20 a $30 \% .^{5}$

Cuando hay autoanticuerpos pueden observarse úlceras orales, petequias, equimosis, gingivitis marginal y artritis de la articulación

Cuadro 1. Criterios de clasificación para el lupus eritematoso generalizado del Colegio Americano de Reumatología

\begin{tabular}{|c|c|}
\hline Eritema malar & Eritema fijo, plano o elevado sobre las eminencias malares que respeta los surcos nasolabiales. \\
\hline Eritema discoide & $\begin{array}{l}\text { Placas elevadas, eritematosas con escama queratósica adherente y taponamiento folicular, } \\
\text { posiblemente cicatrices atróficas en lesiones antiguas. }\end{array}$ \\
\hline Fotosensibilidad & $\begin{array}{l}\text { Exantema cutáneo como resultado de una reacción inusual a la luz solar determinado por } \\
\text { la historia del paciente o por observación física. }\end{array}$ \\
\hline Úlceras orales & Ulceraciones orales o nasofaríngeas, generalmente indoloras, observadas por el médico. \\
\hline Artritis & $\begin{array}{l}\text { Artritis no erosiva que involucra dos o más articulaciones periféricas; caracterizada por } \\
\text { edema, sensibilidad o efusión. }\end{array}$ \\
\hline Serositis & $\begin{array}{l}\text { Pleuritis con historia de dolor pleurítico, frote o evidencia de efusión pleural o pericarditis } \\
\text { documentada por electrocardiografía, frote o evidencia de efusión pericárdica. }\end{array}$ \\
\hline Desórdenes renales & Proteinuria persistente $(\mathrm{N} 500 \mathrm{mg} / 24 \mathrm{~h}, 0.5 \mathrm{~g} / \mathrm{d})$ \\
\hline Desórdenes neurológicos & $\begin{array}{l}\text { Convulsiones o psicosis en ausencia de drogas que las provoquen o alteraciones metabólicas } \\
\text { conocidas, cetoacidosis diabética. }\end{array}$ \\
\hline Desórdenes hematológicos & $\begin{array}{l}\text { Anemia con reticulocitosis o leucopenia }\left(4000 / \mathrm{mm}^{3}\right) \text { en dos o más ocasiones. } \\
\text { Linfopenia }\left(1500 / \mathrm{mm}^{3}\right) \text { en dos o más ocasiones. } \\
\text { Trombocitopenia }\left(100000 / \mathrm{mm}^{3}\right) \text { en ausencia de drogas que lo provoquen. }\end{array}$ \\
\hline Desórdenes inmunológicos & $\begin{array}{l}\text { Anticuerpos anti-ADN } \\
\text { Anticuerpos antifosfolípido positivos. } \\
\text { Prueba positiva de anticoagulante lúpico. } \\
\text { Prueba serológica falsa para sífilis que es positiva por al menos } 6 \text { meses. }\end{array}$ \\
\hline Anticuerpos antinucleares & $\begin{array}{l}\text { Título anormal de anticuerpo antinuclear en ausencia de drogas conocidas que pudieran } \\
\text { asociarse con lupus inducido por drogas. }\end{array}$ \\
\hline
\end{tabular}

Fuente: Weiss JE. Pediatric Systemic Lupus Erythematosus. Pediatrics in Review 2012;33:62-74. 
temporomandibular. ${ }^{5-7}$ Las úlceras orales se presentan como primera manifestación de lupus eritematoso generalizado y son un criterio de diagnóstico. ${ }^{6}$

Las manifestaciones estomatológicas debidas al tratamiento inmunodepresor son candidiasis oral, infección por virus del herpes tipo I, mucositis por inmunodepresores, hiperpigmentaciones reversibles en piel y mucosa por el uso prolongado de hidroxicloroquina y síndrome de Sjögren (xerostomía, ardor bucal, disfagia, disgeusia y predisposición a desarrollar caries dental). ${ }^{5-7}$

\section{CONSIDERACIONES ESTOMATOLÓGICAS PARA EL TRATAMIENTO}

El tratamiento estomatológico de los pacientes con lupus eritematoso generalizado debe ser individualizado. El estomatólogo pediatra, en conjunto con el servicio de inmunología, debe instaurar los cuidados antes, durante y después del tratamiento estomatológico para cada paciente $^{8,9}$ (Cuadro 2). Cuando los pacientes son tratados con antiinflamatorios esteroides e inmunodepresores se producirán cambios hematológicos e inmunitarios que afectarán la producción de células de defensa y reparación tisular; ${ }^{8,9}$ es por ello que se indica profilaxis antibiótica con clindamicina $(20 \mathrm{mg} / \mathrm{kg})$ una hora antes del procedimiento odontológico. ${ }^{10}$ Los procedimientos quirúrgicos dentales deben ser evitados en presencia de autoanticuerpos activos, anemia, leucopenia, neutropenia o trombocitopenia; requiriéndose el uso de hemostáticos locales $\mathrm{O}$, en algunos casos, realizar interdisciplinariamente con un medico inmunólogo ajuste de dosis de anticoagulante, transfusiones, administración de antiinflamatorios esteroides (dosis de estrés), factor de crecimiento hematopoyético, entre otros, para reducir la actividad clínica de la enfermedad y restablecer parámetros en biometría hemática. ${ }^{11,12}$
Debe eliminarse cualquier foco infeccioso en la cavidad bucal pues suprime el tratamiento inmunodepresor y predispone a infecciones secundarias. ${ }^{8,9}$ Los fármacos que se prescriban deben ser los que se metabolizan en el hígado como la clindamicina y el acetaminofén; evitando los de excreción renal como penicilinas, cefalosporinas, antimicóticos y antiinflamatorios no esteroides. ${ }^{9,13}$

\section{CASO CLÍNICO}

Niña de 5 años y 11 meses de edad con diagnóstico de lupus eritematoso generalizado, producto de la gesta 4, de padres jóvenes sanos, no consanguíneos pero con antecedentes familiares de lupus. Embarazo normal, producto obtenido a término por vía vaginal, peso de $3300 \mathrm{~kg}$, talla de $51 \mathrm{~cm}$, Apgar 8/9 e hitos del desarrollo normal. Actualmente cursa escolaridad irregular con interacción social deficiente.

Antecedentes de derrame pericárdico, anemia hemolítica, ANA +++, artritis, nefritis, síndrome antifosfolípidos, vasculitis del sistema nervioso central. En tratamiento con trimetoprima/ sulfametoxazol, furosemida, captopril, ácido ursodeoxicólico, omeprazol, leche de aluminio y magnesio, dexametasona, acenocumarol, levetiracetam, gammaglobulina, rituximab, ciclofosfamida y pulsos de metilprednisolona.

Ingresó al Instituto Nacional de Pediatría en muy malas condiciones generales, con desnutrición crónica, aspecto desalineado, caída abundante de cabello, datos de respuesta inflamatoria sistémica: temperatura de $39.9^{\circ} \mathrm{C}$, FC 180 lpm, FR 40 x', TA 108/58. Clínicamente reactiva, indiferente al medio externo, respondía a estímulos dolorosos, palidez de tegumentos $+/+++$, adecuado estado de hidratación, cráneo sin endo- ni exostosis, movimientos oculares espontáneos, narinas permeables con moco blanquecino, cornetes hiperémicos, cavidad oral con mucosas 
Gómez-Contreras P et al. Tratamiento estomatológico interdisciplinario del lupus

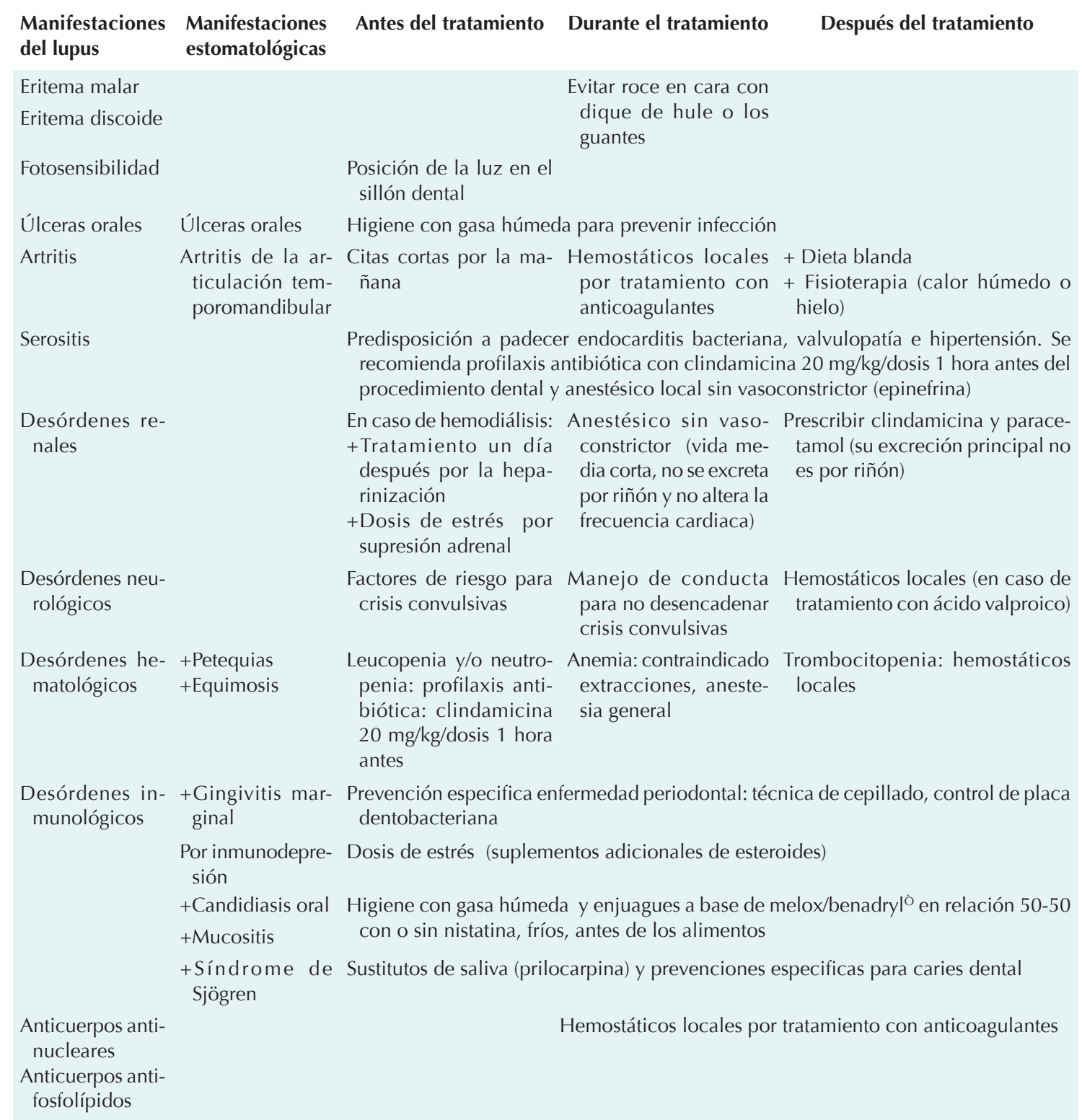

Fuente: Rossi SS, Glick M. Lupus erythematosus: consideration for dentistry. JADA 1998;129:330-339 (modificada).

íntegras, bien hidratadas con cambio de color a eritematoso y salida de material purulento en fondo de saco correspondiente a órgano dentario 51; pérdida de tejido amelodentinario en 18 órganos dentarios de 20 presentes. Faringe con hiperemia $+/+++$, amígdala Gll con descarga retronasal blanquecina, cuello con pulsos carotídeos palpables, sin megalias. Tórax con aumento de trabajo respiratorio a expensas de tiraje subcostal leve y polipnea de $40 \mathrm{rpm}$, campos pulmonares con estertores crepitantes de predominio basal bilateral. Precordio normodi- 
námico, con ruidos disminuidos de intensidad en mesocardio sin agregados. Abdomen con peristaltismo en los cuatro cuadrantes con adecuada intensidad y frecuencia, blando, depresible sin dolor a la palpación media ni profunda. Genitales externos fenotípicamente femeninos, Tanner P-I, G-I. Extremidades simétricas y eutróficas, Ilenado capilar inmediato, codos con lesiones eritematosas pruriginosas tipo úlceras dolorosas que limitan el movimiento. Hematológicamente ha cursado con anemia hemolítica; en la última biometría hemática se reportó hemoglobina $8.2 \mathrm{~g} / \mathrm{dL}$, leucocitos $8700 / \mathrm{mm}^{3}$, neutrófilos 800 / $\mathrm{mm}^{3}$, plaquetas $490000 / \mathrm{mm}^{3}$.

El servicio de inmunología solicitó valoración de la cavidad oral debido a la presencia de focos infecciosos, absceso en incisivo central y múltiples caries. Se diagnosticó caries temprana severa de la infancia, definida por la Asociación Americana de Odontopediatría como índice ceo (cariado, extraído por caries y obturado), en niños de 5 años, mayor a 6 órganos dentarios afectados $^{14}$ (Figura 1).

Se indica rehabilitación bucal bajo anestesia general por la extensión del tratamiento, corta edad, ser un paciente inmunocomprometido y porque los focos infecciosos interfieren con el tratamiento inmunodepresor. ${ }^{15}$ Se informó al

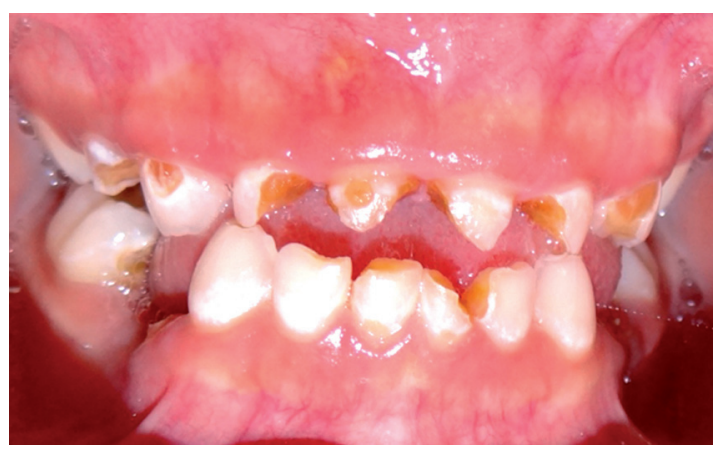

Figura 1. Focos infecciosos en la cavidad oral (caries temprana de la infancia). servicio de inmunología que se haría un tratamiento estomatológico radical, con múltiples extracciones, por lo que se hicieron las siguientes consideraciones:

1. Interconsulta con el servicio de cardiología por el antecedente de derrame pericárdico.

2. Administración de un pulso de metilprednisolona $30 \mathrm{mg} / \mathrm{kg} / \mathrm{dosis}$.

3. Administración de gammaglobulina intravenosa $1 \mathrm{~g} / \mathrm{kg} /$ dosis.

4. Transfusión de un paquete globular $15 \mathrm{mg} / \mathrm{kg} /$ día.

5. Biometría hemática de control, antes de la rehabilitación bucal, para corregir la anemia hemolítica.

6. Dosis de estrés $(50 \mathrm{mg} / \mathrm{kg} /$ dosis $)$ con hidrocortisona pre- y posrehabilitación bucal.

7. Tratamiento farmacológico terapéutico con clindamicina (30 mg/kg/día) durante siete días, así como analgésico tipo ibuprofeno $(10 \mathrm{mg} / \mathrm{kg} /$ dosis $)$ durante tres días.

El objetivo de estas medidas fue reducir la actividad clínica de la enfermedad, aumentar la hemoglobina a más de $10 \mathrm{~g} / \mathrm{dL}$ y obtener una adecuada función plaquetaria y de hemostasia. Como resultado se obtuvieron $13.5 \mathrm{~g} / \mathrm{dL}$ de hemoglobina, tiempo de protrombina 9.6 segundos, tiempo de tromboplastina parcial 18.9 segundos e INR de 0.8 . Se realizó la extracción de nueve órganos dentarios infectados quedando la paciente libre de procesos infecciosos (Figura 2).

\section{DISCUSIÓN}

Al tratamiento estomatológico del paciente con lupus eritematoso generalizado lo influyen la actividad clínica de la enfermedad y su trata- 


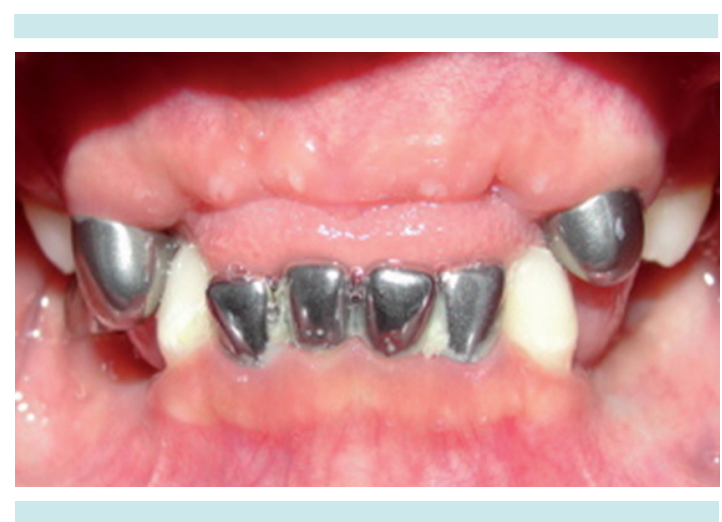

Figura 2. Paciente libre de focos infecciosos.

miento médico. Durante episodios agudos no se podrá realizar ningún tratamiento estomatológico, salvo que se trate de una urgencia hemorrágica o infecciosa y que el paciente se encuentre hospitalizado. ${ }^{9}$ Durante las fases crónica o estable de la enfermedad el estomatólogo deberá preguntar al inmunólogo acerca de daño renal, cardiaco, neurológico, hematológico y acerca del tratamiento médico para que, interdisciplinariamente, se establezcan los cuidados antes, durante y después del tratamiento dental. ${ }^{8}$ Los procedimientos dentales quirúrgicos se realizarán bajo profilaxis antibiótica; los procedimientos simples con ayuda de hemostáticos locales y los mayores, como rehabilitación bucal bajo anestesia general o extracción de terceros molares, se realizarán intrahospitalariamente. ${ }^{8,9}$

En los pacientes inmunocomprometidos las infecciones de origen dental afectan el éxito del tratamiento inmunodepresor, lo que predispone a infecciones sistémicas. ${ }^{9}$ Ángel y Albilia señalaron que las infecciones de origen dental y algunas úlceras orales son asintomáticas, debido al uso crónico de antiinflamatorios esteroides y no esteroides, y recomendaron la prevención específica y el seguimiento estrecho. ${ }^{9,7}$

Rossi y Brennan informaron que 7.5 a $30 \%$ de los pacientes con lupus eritematoso generalizado padecen síndrome de Sjögren que disminuye el flujo salival y predispone a tener caries. El estomatólogo pediatra deberá realizar pruebas de permeabilidad de las glándulas salivales, medidas higiénicas, dietéticas, preventivas y restaurativas de la cavidad oral. ${ }^{8,5}$

La importancia de reportar este caso clínico consiste en informar las modificaciones interdisciplinarias que se realizan en el tratamiento estomatológico de los pacientes con lupus eritematoso generalizado pues se cuenta con muy poca información al respecto.

\section{REFERENCIAS}

1. Berrón PR. Enfermedades Autoinmunitarias en el niño. En: Berrón PR, (ed). Lupus Eritematoso Sistémico. Ciudad de México: Editorial Alfil; 2007;19-43.

2. Weiss JE. Pediatric Systemic Lupus Erythematosus. Pediatrics in Review 2012;33:62-74.

3. Levy DM, Kamphuis S. Systemic Lupus Erythematosus in Children and Adolescents. Pediatr Clin N Am 2012;59:345364.

4. Habibi S, Saleem MA, Ramanan AV. Juvenile Systemic Lupus Erythematosus: Review of Clinical Features and Management. Indian pediatrics 2011;48:879-87.

5. Brennan MT, Valerin MA, Napeñas JJ, Lockhart PB. Oral manifestations of patients with lúpus erythematosus. Dent Clin N Am 2005;49:127-141.

6. López $L$, , Moret Y, Dorrego M, Henning M. Manifestaciones bucales del lupus eritematoso. Revisión de la literatura. Acta odontológica venezolana 2007;45:1-7.

7. Ángel N, Echeverry N, Restrepo P, González L, Rodríguez L, Vásquez $\mathrm{G}$. Oral manifestations in patients with systemic lúpus erythematosus. Rev Colomb Reumatol 2010;17:1321.

8. Rossi SS, Glick M. Lupus erythematosus: consideration for dentistry. JADA 1998;129:330-339.

9. Albilia JB, Clokie CML, Sándor GKB. Systemic lúpus erythematosus: a review for dentists. JCDA 2007;73:823-28.

10. Wilson W, Taubert KA, Gewitz M, Lockhart PB, Larry MB, Levison M, et al. Prevention of Infective Endocarditis: Guidelines from the American Heart Association Rheumatic, Fever, Endocarditis, and Kawasaki Disease Committee, Council on cardiovascular Disease in the Young, and the Council on clinical cardiology, Council on Cardiovascular Surgery and Anesthesia, and the Quality of Care and Outcomes Research Interdisciplinary Working Group. Circulation 2007;116:1736-1754. 
11. Castellanos SJ, Diaz GL, Gay ZO. Medicina en Odontologia. Manejo dental de pacientes con enfermedades sistémicas. En: Castellanos SJ, Diaz GL, Gay ZO,(ed). Enfermedades inmunitarias. México: Manual Moderno 2002;191-219.

12. Little JW, Falace DA. Tratamiento odontológico del paciente bajo tratamiento médico. En: Little JW, Falace DA, (ed). Artritis. España: Editorial Harcourt Brace 1998;357-372.

13. Castellanos SJ, Diaz GL, Gay ZO. Medicina en Odontologia. Manejo dental de pacientes con enfermedades sistémi- cas. En: Castellanos SJ, Diaz GL, Gay ZO, (ed). Alteraciones renales. México: Editorial manual moderno; 2002;103120.

14. Policy on early childhood caries (ECC): Classifications, Consequences, and Preventive strategies. Pediatr Dent 2012-2013,34: 50-52.

15. De la Teja AE, Cadena GA, Elías MG, Estrada HE, Escudero CA, Isunza RA. Rehabilitación bucal en niños bajo anestesia general. Acta Pediatr Mex 2004;25:74-80.

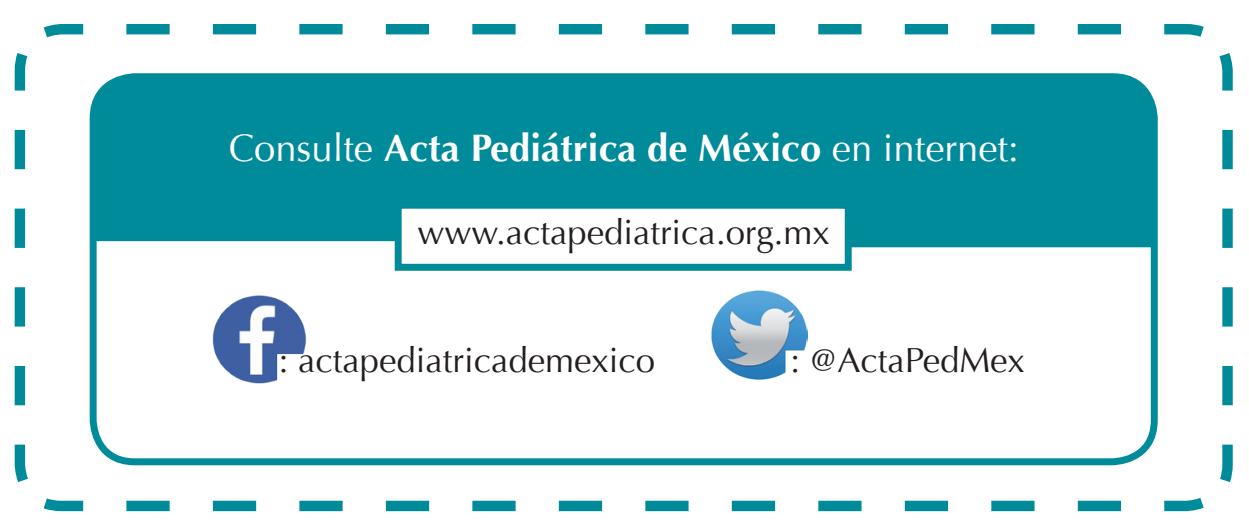

